\title{
Characterization of GaAs:Cr sensors using the charge-integrating JUNGFRAU readout chip
}

D. Greiffenberg ${ }^{1}$, M. Andrä ${ }^{1}$, R. Barten ${ }^{1}$, A. Bergamaschi ${ }^{1}$, P. Busca ${ }^{2}$, M. Brückner ${ }^{1}$, S. Chiriotti Alvarez ${ }^{1}$, I. Chsherbakov $^{3}$, R. Dinapoli ${ }^{1}$, P. Fajardo ${ }^{2}$, E. Fröjdh ${ }^{1}$, C. Lopez-Cuenca ${ }^{1}$, A. Lozinskaya ${ }^{3}$, M. Meyer ${ }^{1}$, D. Mezza ${ }^{1}$, A. Mozzanica $^{1}$, S. Redford ${ }^{1}$, M. Ruat ${ }^{2}$, Ch. Ruder ${ }^{1}$, B. Schmitt ${ }^{1}$, X. Shi ${ }^{1}$, D. Thattil ${ }^{1}$, G. Tinti ${ }^{1}$, O. Tolbanov $^{3}$, A. $^{2}$ Tyazhev $^{3}$, S. Vetter ${ }^{1}$, A. Zarubin ${ }^{3}$, J. Zhang ${ }^{1}$

${ }^{1}$ Paul Scherrer Institut (PSI), CH-5232 Villigen PSI, Switzerland

${ }^{2}$ European Synchrotron Radiation Facility, F-38000 Grenoble, France

${ }^{3}$ Tomsk State University (TSU), Tomsk, 634050, Russia

\section{Abstract}

Chromium compensated GaAs sensors have been characterized using the charge-integrating readout chip JUNGFRAU. Due to its low noise performance and $75 \times 75 \mu^{2}$ pixel size, JUNGFRAU enables a precise measurement of the charge (of either polarity) with a high spatial resolution.

Several sensor parameters like dark current, noise and spectral performance as well as the charge transport properties of the electrons have been determined. The short lifetime of holes in GaAs:Cr gives rise to an effect where pixels adjacent to a pixel with a photon hit show a strong negative signal when being absorbed close to the readout electrode. This so-called 'crater effect' has been simulated and allows an estimation of the hole lifetime in GaAs:Cr.

\section{$\underline{\text { Introduction }}$}

The most common sensor material for detectors at synchrotron sources and free electron lasers is silicon due to its outstanding material quality in terms of uniformity and charge carrier transport properties. However, the low atomic number of silicon $(Z=14)$ is the reason for a relatively low absorption efficiency for photon energies above $20 \mathrm{keV}$. Sensors with high atomic number, so-called high-Z sensors, provide absorption efficiencies that are significantly higher in this energy range. Some of most commonly used high-Z sensors for photon detection are GaAs and CdTe. While the absorption efficiency of silicon drops below $50 \%$ for photon energies above 18 keV, GaAs keeps this efficiency up to photon energies of $55 \mathrm{keV}$ and CdTe until $104 \mathrm{keV}^{1}$ [1]. Compared to silicon, high-Z sensors are (still) lacking in several aspects: homogeneity, charge transport properties, charge trapping (leading to polarization effects), long ranged fluorescence photons, and others [2],[3],[4],[5]. However, several authors report that the quality has improved over the last years [6],[7],[8],[9].

Compared to $\mathrm{CdTe}$, GaAs has some advantages: better mechanical stability and lower energy fluorescence photons $\left(E_{G a ~ K \alpha 1}=9.25 \mathrm{keV}, \mathrm{E}_{\mathrm{As} K \alpha 1}=10.54 \mathrm{keV}\right.$ vs. $\left.\mathrm{E}_{\mathrm{Cd} \mathrm{K \alpha 1}}=23.17 \mathrm{keV}, \mathrm{E}_{\mathrm{Te} K \alpha 1}=27.47 \mathrm{keV}\right)$, thus having a shorter absorption length for reabsorption in the sensor $\left(\mathrm{d}_{\mathrm{Ga} K \alpha 1}=40.2 \mu \mathrm{m}, \mathrm{d}_{\mathrm{As} K \alpha 1}=15.6 \mu \mathrm{m}\right.$ vs. $\mathrm{d}_{\mathrm{Cd} \mathrm{K}_{1} 1}=118.7 \mu \mathrm{m}, \mathrm{d}_{\mathrm{Te}} \mathrm{K \alpha 1}=62.4 \mu \mathrm{m}$ ) [10],[11]. Moreover, no polarization effects have been reported so far [2],[9]. On the other hand, the use of semiinsulating GaAs sensors was already discussed in the 1960s, but was not pursued due to shortcomings in spatial and temporal stability of the signals [12]. In 2002, the Tomsk State University (TSU) presented a novel method to create high-resistivity GaAs sensors by using chromium as a dopant and first results using it as sensor material in 2011 were promising [13],[14],[15].

The motivation of this study is the characterization of GaAs:Cr sensors using charge-integrating readout chips and to get an understanding of the usability of $\mathrm{GaAs}: \mathrm{Cr}$ sensors for synchrotron applications. Compared to classical single-photon counting chips, the use of charge-integrating readout chips allows the precise measurement of charge of either polarity collected during a pre-defined integration time potentially giving a better insight into the sensor behavior.

\footnotetext{
${ }^{1}$ Typical sensor thicknesses used for the calculations: Silicon: $500 \mu \mathrm{m}$ / GaAs: $500 \mu \mathrm{m}$ / CdTe: $750 \mu \mathrm{m}$
} 


\section{GaAs:Cr sensor}

TSU produces the GaAs:Cr sensors from commercially available low resistivity n-type GaAs wafers which are postprocessed to achieve the high resistivity by diffusing chromium into the GaAs wafer. The wafers used in this paper where produced in 2016 and the growth method was LEC (Liquid Encapsulated Czrochalski). After the chromium diffusion process the wafers are thinned down to a thickness of $516 \mu \mathrm{m}$. The pixel contacts (with a pixel pitch of $75 \times 75 \mu \mathrm{m}^{2}$ ) as well as the backside contact are made from $1.5 \mu \mathrm{m}$ nickel.

Bumpbonding of the sensors to the readout chip was done at the Paul Scherrer Institut (PSI) using a standard indium bumpbonding process.

\section{Readout chip}

The readout chip used for this study was the charge-integrating JUNGFRAU ASIC (Application Specific Integrated Chip) with a pixel pitch of $75 \times 75 \mu^{2}$ which was developed at PSI primarily for the SwissFEL free electron laser accelerator [16][17]. The pixel array consists of $256 \times 256$ pixels resulting in an active area of $19.2 \times 19.2 \mathrm{~mm}^{2}$.

A common feature of the charge integrating readout chips developed at PSI is the dynamic gain adjustment in the preamplifier which allows a low noise operation in a low photon flux environment (=high gain) and a maximum dynamic range, albeit with a higher noise, of $10^{4} \times 12.4 \mathrm{keV}^{2}$ in a high flux environment [17]. This is achieved by having three different feedback capacitors in the preamplifiers feedback loop which are automatically switched depending on the number of incoming photons ${ }^{3}$. However, this automatic gain switching feature was developed to work only in hole collection mode. As the collection of electrons is preferred in case of high-Z sensors, the gain has to be statically selected before each acquisition. In this study the gain was fixed to high gain (corresponding to "GO" in [16],[17]), i.e. the maximum dynamic range was around $240 \mathrm{keV}$ and the noise performance (83 \pm 6$)$ e- ENC (r.m.s.) in the case of silicon sensors and an integration time of $10 \mu \mathrm{s}$ [17].

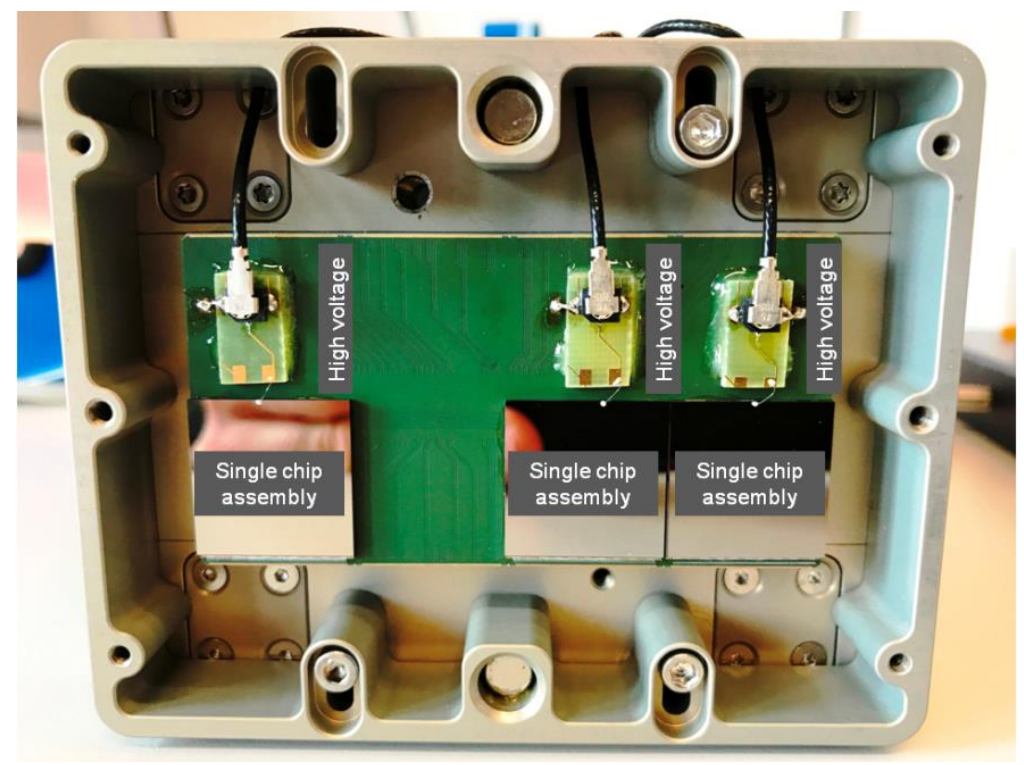

Figure 1: Example of a hybrid module consisting of three single chip assemblies (here: three times CdTe senors). The single chip assemblies can be chosen in arbitrary combinations. For the measurements of this paper, different modules with $\mathrm{GaAs}: \mathrm{Cr}$, CdTe and silicon sensors were used. Currently, single chip assemblies with GaAs, CdTe, CdZnTe and silicon sensors bumpbonded to JUNGFRAU ASICs are available. Each high-Z assembly is individually biased by an external high voltage source. Assemblies with silicon sensor are biased by the readout system (not present in this example).

\footnotetext{
${ }^{2}$ In case of JUNGFRAU, AGIPD and GOTTHARD readout chips.

${ }^{3}$ The electronic noise is lower than the Poissonian fluctuations at each point of the dynamic range in case of dynamic gain switching, ensuring the same data quality as single photon counting systems [17].
} 
The test systems are based on a standard JUNGFRAU multi-ASIC module. In the standard silicon version 8 ASICs are bumpbonded in a $4 \times 2$ arrangement on a single silicon sensor. In this study, single chip assemblies (single chip sized sensors bumpbonded to single ASICs) are mounted on the standard readout board [Fig. 1]. The high voltage of each sensor is supplied individually by external high voltage sources (Keithley 2410/Keithley 6487), except in the case of silicon sensors, where the high voltage is generated by the readout system itself. In order to be able to use hybrid modules with high-Z sensors and silicon sensors at the same time in parallel, thus collecting electrons in the high-Z sensors and holes in the silicon sensor, the working point of the preamplifier has been set to the middle of the dynamic range providing the same dynamic range for charge carriers of each polarity, but reduced by a factor of 2 (with respect to a single polarity operation).

The hybrid modules are readout by a JUNGFRAU Module Control Board (JMCB) developed at PSI [16]. The maximum achievable frame rate is $1 \mathrm{kHz}$ using a single $10 \mathrm{GBit}$ link, however most commonly the system was read out at a rate of $500 \mathrm{~Hz}$. The metal frame holding the JMCB contains cooling channels which were used to stabilize the temperature of the single chips by cooling them from the backside. If not stated otherwise, the operating temperature of the water was set to $+15^{\circ} \mathrm{C}$.

\section{$\underline{\text { Results }}$}

\section{Gain and noise}

A gain calibration was performed using monochromatic $60 \mathrm{keV}$ photons at the BM05 beamline at the ESRF [18]. The photon flux was adjusted to have clearly separated photon hits, which resulted in having roughly 200 photon hits per frame or a photon flux of $2.7 \cdot 10^{5}$ photons per $\mathrm{mm}^{2}$ and second (for an integration time of $2 \mu \mathrm{s}$ ). The sensor bias voltage was $-300 \mathrm{~V}$.

The results from the gain calibration are shown in Fig. 2. An example of a single pixel spectrum is shown in Fig. 2, left. The photopeak was fitted with a Gaussian with a relatively tight fit range on the lower energy side of $-0.5 \cdot \sigma$. $(\sigma$ was estimated from fitting the higher energy shoulder of the photopeak). The mean gain is 33.75 ADU/keV with a dispersion of $1.11 \mathrm{ADU} / \mathrm{keV}$ or $3.29 \%$ (r.m.s.). (The gain dispersion of single chip assemblies with silicon sensor is typically smaller with values between 1.6-2.0\%).
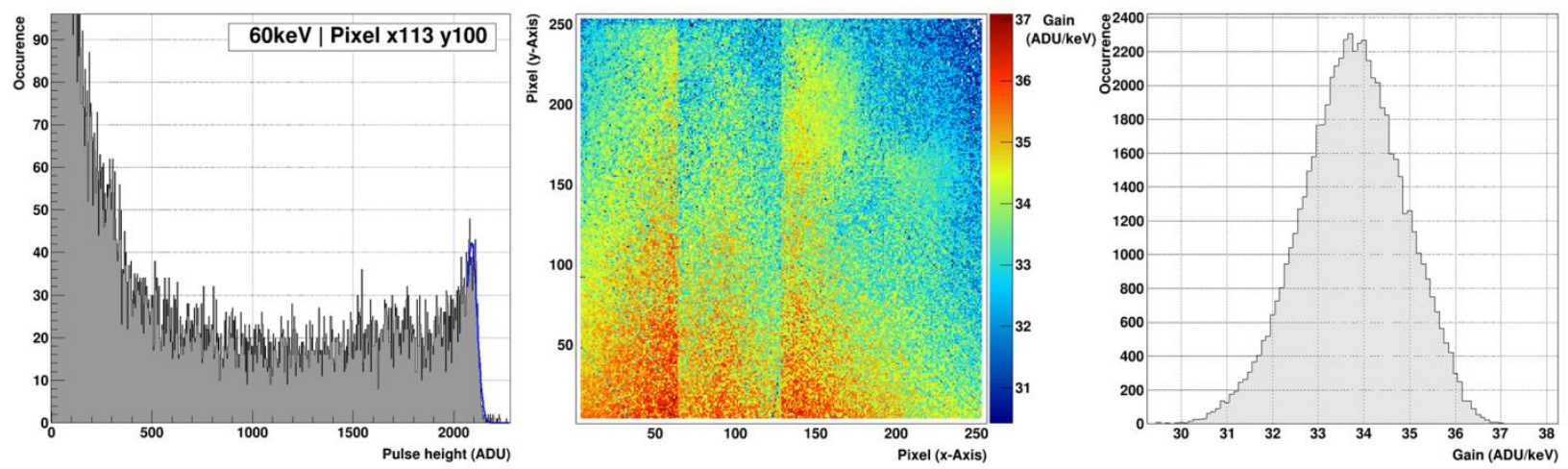

Figure 2: (left) Example of a single pixel spectrum of $60 \mathrm{keV}$ monochromatic photons. The photopeak was fitted with a Gaussian function to determine the gain (ADU/keV). (middle/right) Map and distribution of the gain (ADU/keV).

The noise performance of the system was evaluated (Fig. 3) by fitting the distribution of the pixel output without photon signal with a Gaussian. The peak position of this distribution of dark frames will be later referred to as pedestal, the dispersion (r.m.s.) corresponds to the noise of a pixel in units of ADU and is converted in ENC using

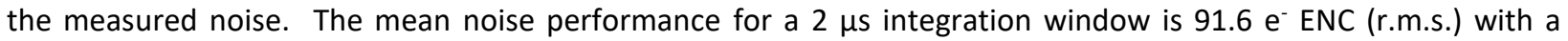
dispersion of $6.0 \%$. The noise level worsens with increasing integration time mainly due to the increased dark current contribution, reaching 115.9 e- ENC (r.m.s.) with a dispersion of $5.5 \%$ for a 5 us integration window. In 
comparison, the noise performance of a single chip assembly with silicon sensor gives a mean value of $82.4 \mathrm{e}^{-}$ENC (r.m.s.) with a dispersion of $6.4 \%$ (Integration time of $2 \mu \mathrm{s}$ ).
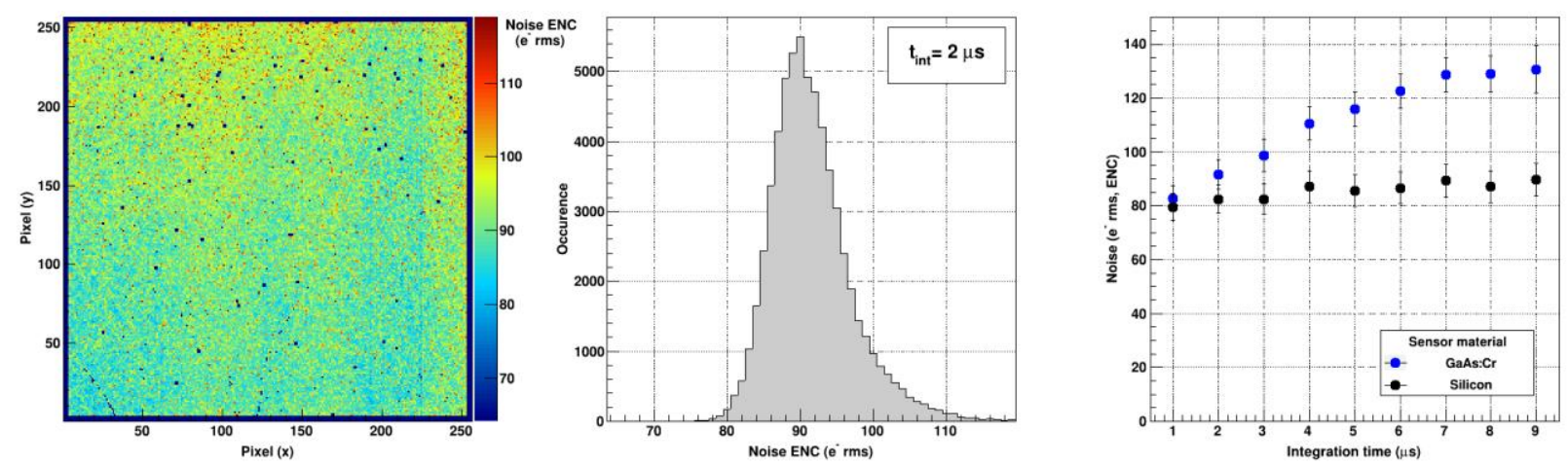

Figure 3: (left/middle) Map and distribution of the noise performance of the GaAs:Cr assembly for an integration time of $2 \mu \mathrm{s}$ $\left(\mathrm{U}=-300 \mathrm{~V} / \mathrm{T}=+15^{\circ} \mathrm{C}\right)$. (right) Mean noise performance as function of the integration time, comparing an assembly with GaAs:Cr and silicon sensor. Note: For integration times above $7 \mu \mathrm{s}$, the dynamic range of several pixels of the GaAs: $\mathrm{Cr}$ sensor reached saturation, thus not showing an increased noise level.

\section{Dark current and dynamic range}

In order to define reasonable operating parameters in terms of integration time and working point of the preamplifier (as mentioned earlier), the dark current was determined. The bias voltage of the sensor was set to $\mathrm{U}=-300 \mathrm{~V}$ and the integration time was increased in steps of $1 \mu \mathrm{s}$, starting with a minimum integration time of $1 \mu \mathrm{s}$. A scan of the integrated charge as function of the integration time of an arbitrary pixel can be seen in Fig.2, left. The dark current for each pixel was extracted by performing a linear fit from $1 \mu \mathrm{s}$ to $4 \mu \mathrm{s}$. The resulting dark current in units of $A D U / k e V$ can be translated into $\mathrm{pA}$ by making use of equation 1 :

Equation 1: (1) Conversion by dividing using the pixel gain (ADU/keV) (2) Conversion into number of charge carriers $n$ by using the electron-hole pair creation energy of $4.2 \mathrm{eV}$ per electron-hole pair [2] (3) Conversion into current by converting into $\mathrm{C} / \mathrm{s}=\mathrm{A}$.

$$
\frac{A D U}{\mu s} \stackrel{1}{\rightarrow} \frac{k e V}{\mu s} \stackrel{2}{\rightarrow} \frac{n}{\mu s} \stackrel{3}{\rightarrow} A
$$

Figure 4 (right) shows the distribution of the dark current for all pixels. The average dark current was $(370.6 \pm 0.2)$ pA per pixel with a dispersion of $(55.9 \pm 0.1)$ pA (r.m.s.). The overall dark current through all pixels $(256 \times 256$ pixels) was $25.1 \mu \mathrm{A}$, which results in a resistivity of the GaAs:Cr sensor of $\rho=8.5 \cdot 10^{8} \Omega \cdot \mathrm{cm}^{4}$.

\footnotetext{
${ }^{4}$ The resistivity has been evaluated with the following formula:

$$
\rho=\frac{U}{I} \cdot \frac{A}{d}
$$

U: Sensor bias voltage, I: Current through all pixels of the sensor, A: Sensor area, d: Sensor thickness
} 

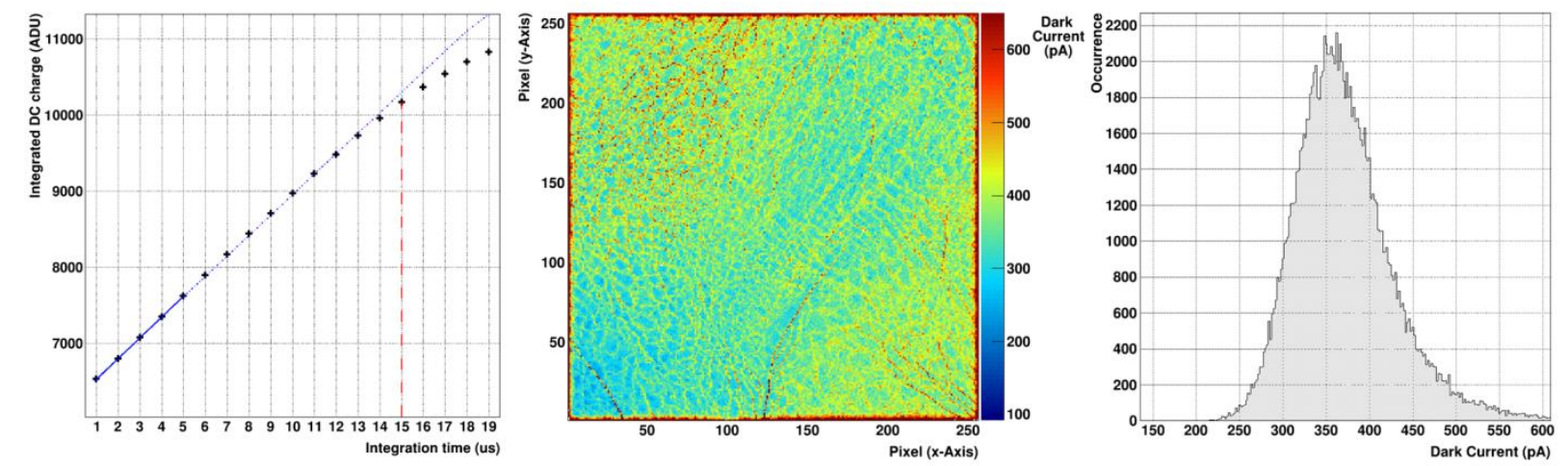

Figure 4: (left) Integrated charge as function of the integration time for a bias voltage of $-300 \mathrm{~V}$ and at $\mathrm{T}=+15^{\circ} \mathrm{C}$ for an arbitrary pixel. (center) Map of the dark current per pixel (pA). (right) Distribution of the dark current over the pixel matrix. The mean value is $391.0 \mathrm{pA}$ with a dispersion of $65.9 \mathrm{pA}$ (r.m.s.).

The overall current from the high voltage supply (Keithley 6487) was $37 \mu \mathrm{A}$, indicating that roughly $32 \%$ of the current was flowing over the edge of the sensor into the guard ring. As can be seen in the dark current map Fig. 4 (center), the current in the edge pixels is significantly higher than for the rest of the pixel matrix, reducing the dynamic range close to the sensor boundaries: The outermost pixels draw an average current of $840.1 \mathrm{pA} / \mathrm{pixel}$ $(+115 \%)$, the average current through pixels in the $2^{\text {nd }}$ row/column is $551.7 \mathrm{pA} / \mathrm{pixel}(+41 \%)$ and $429.2 \mathrm{pA} / \mathrm{pixel}$ $(+10 \%)$ for pixels in the $3^{\text {rd }}$ row/column.

The maximum integration time per pixel was extracted by evaluating the integration time, at which the deviation between extrapolated linear fit and the scan of the integrated charge as function of the integration time exceeds 1 $\%$. The mean maximum integration time is $13.48 \mu$ s with a dispersion of $2.06 \mu$ s (r.m.s.).

The maximum measurable signal per pixel is calculated by taking the difference between the value of the integrated DC current at the maximum integration time and the pedestal and normalizing it by the gain of each pixel. The results are summarized in Fig. 5. The maximum measurable signal is $(109.04 \pm 0.03) \mathrm{keV}$ with a dispersion of $7.0 \mathrm{keV}$ (r.m.s.).
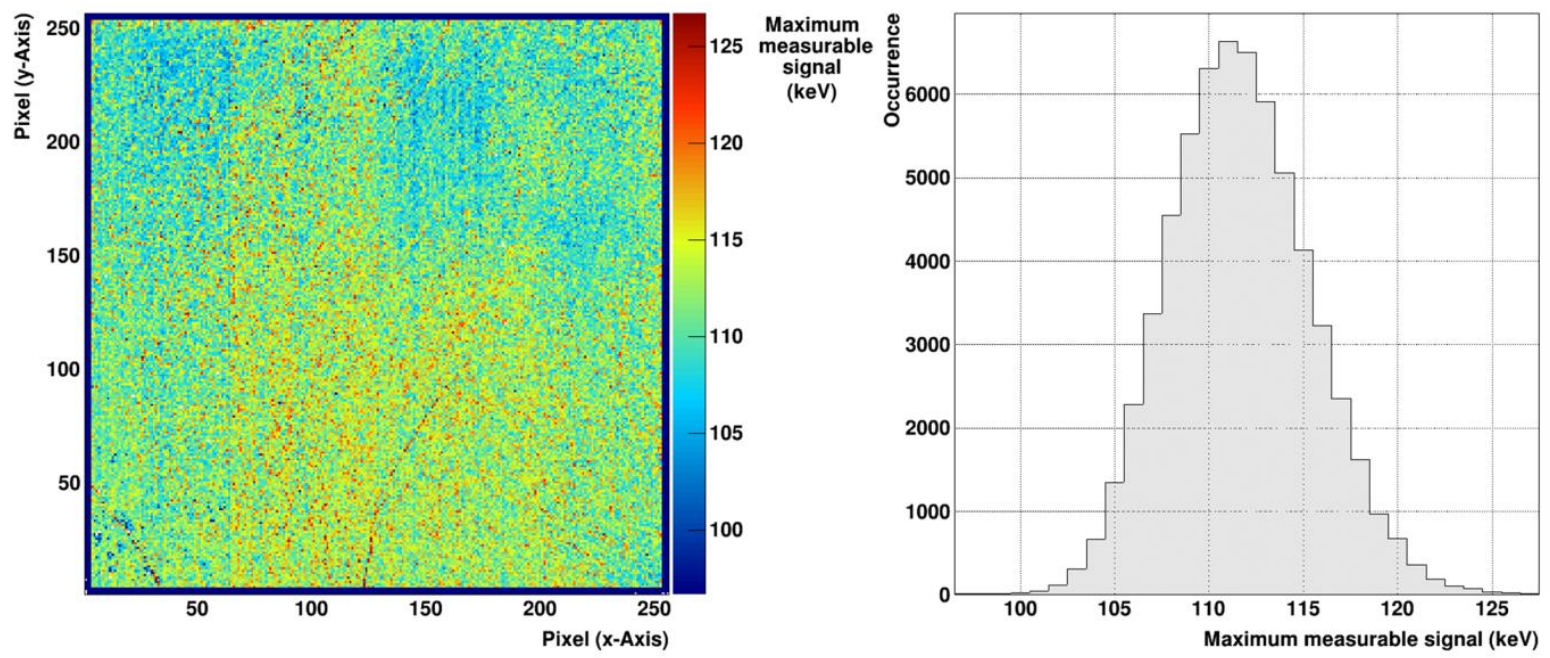

Figure 5: (left) Map of the maximum measurable signal (in keV) and (right) distribution of the maximum measurable signal (in keV).

At a temperature of $+15{ }^{\circ} \mathrm{C}$ the available dynamic range is reduced by the dark current at a rate of $8.45 \mathrm{keV} / \mu \mathrm{s}$ which as a consequence means that the integration time has to be set short enough to allow for enough residual dynamic range. For the experiments the integration time was set to $2 \mu$ s leaving a dynamic range of $92.1 \mathrm{keV}$. 
Previous measurements showed that the dark current was reduced by a factor of 9 when cooling down to $-12{ }^{\circ} \mathrm{C}$ at the cost of measures to prevent condensation (mechanical housing with a nitrogen saturated environment). Moreover, for a regular detector which would collect only one polarity of charge carriers the dynamic range would roughly double.

\section{Spectral capabilities}

The spectral capabilities were evaluated by illuminating the system with monochromatic $60 \mathrm{keV}$ photons at the BM05 beamline at the ESRF [18]. The photon flux was low enough to ensure clearly separated photon hits. The data was pedestal and gain corrected and then treated with a photon finder algorithm. The photon finder identifies pixels with a signal five times higher than the noise level and extracts pixel clusters of different sizes: $1 \times 1,3 \times 3$ and $5 \times 5$ clusters are centered around the pixel with the highest signal, $2 \times 2$ clusters are selected according to the highest cluster sum. (The three outermost pixel rows/columns were not treated in this analysis as the aforementioned clustering method could otherwise result in incomplete clusters.)

Fig. 6 shows the $60 \mathrm{keV}$ energy spectra using the whole $256 \times 256$ pixel matrix, except masked pixels. The criteria to mask pixels were a noise performance which exceeds ten times the r.m.s. of the noise distribution and/or a baseline that runs out of limits. Typically less than $1 \%$ of the pixels are excluded by these criteria. The height of the photopeak using only the signal information of the central pixel $(=1 \times 1$ cluster | gray spectrum) only reaches around $35 \%$ of the $2 \times 2$ pixel cluster spectrum (red spectrum) which indicates a high level of charge sharing between pixels. The fluorescence photon escape peaks at around $50 \mathrm{keV}$ arise from fluorescence photons from $\mathrm{Ga}$ and As which are either absorbed in neighboring pixels or escape out of the sensor. Due to the short range ( $d_{G a} K_{\alpha 1}=40 \mu \mathrm{m} / d_{A s} K_{\alpha 1}=16 \mu \mathrm{m}$ ) and relatively low energy ( $E_{G a} K_{\alpha 1}=9.25 \mathrm{keV} / E_{A s}$ $\mathrm{k} \alpha 1=10.54 \mathrm{keV}$ ) of the fluorescence photons, no fluorescence photon peak is visible in any spectra $(1 \times 1 / 2 \times 2 / 3 \times 3 / 5 \times 5)$. In the gray spectrum the cut-off criteria of the cluster finder corresponding to five times the noise at around $2 \mathrm{keV}$ is clearly visible. The tail from the noise peak reaches into the spectrum at low energies and the charge sharing between 4 pixels generates energies around $15 \mathrm{keV}$. The shoulder in the gray spectrum at around half the incoming photon energy at $30 \mathrm{keV}$ arises from the photon finder which always centers the cluster around the pixel with the highest signal.

The FWHM of the $60 \mathrm{keV}$ photopeak using $2 \times 2$ clusters is $4.14 \mathrm{keV}$ or $6.9 \%$. It is difficult to put this result in perspective given the fact that a $256 \times 256$ pixel detector with relatively small pixels $\left(75 \times 75 \mu \mathrm{m}^{2}\right)$ was used. Results with a single photon counting Medipix3RX device having a pixel pitch of $55 \mu \mathrm{m}$ with charge summing mode report a FWHM of $16.2 \%$ in high gain mode, when using the photopeak of a ${ }^{241} \mathrm{Am}$ source at $59.5 \mathrm{keV}$ [2]. The FWHM of $60 \mathrm{keV}$ measured with the spectroscopic imaging readout chip HEXITEC with a $250 \mu \mathrm{m}$ pixel pitch ranges between $2.8-3.3 \mathrm{keV}[19]$. 


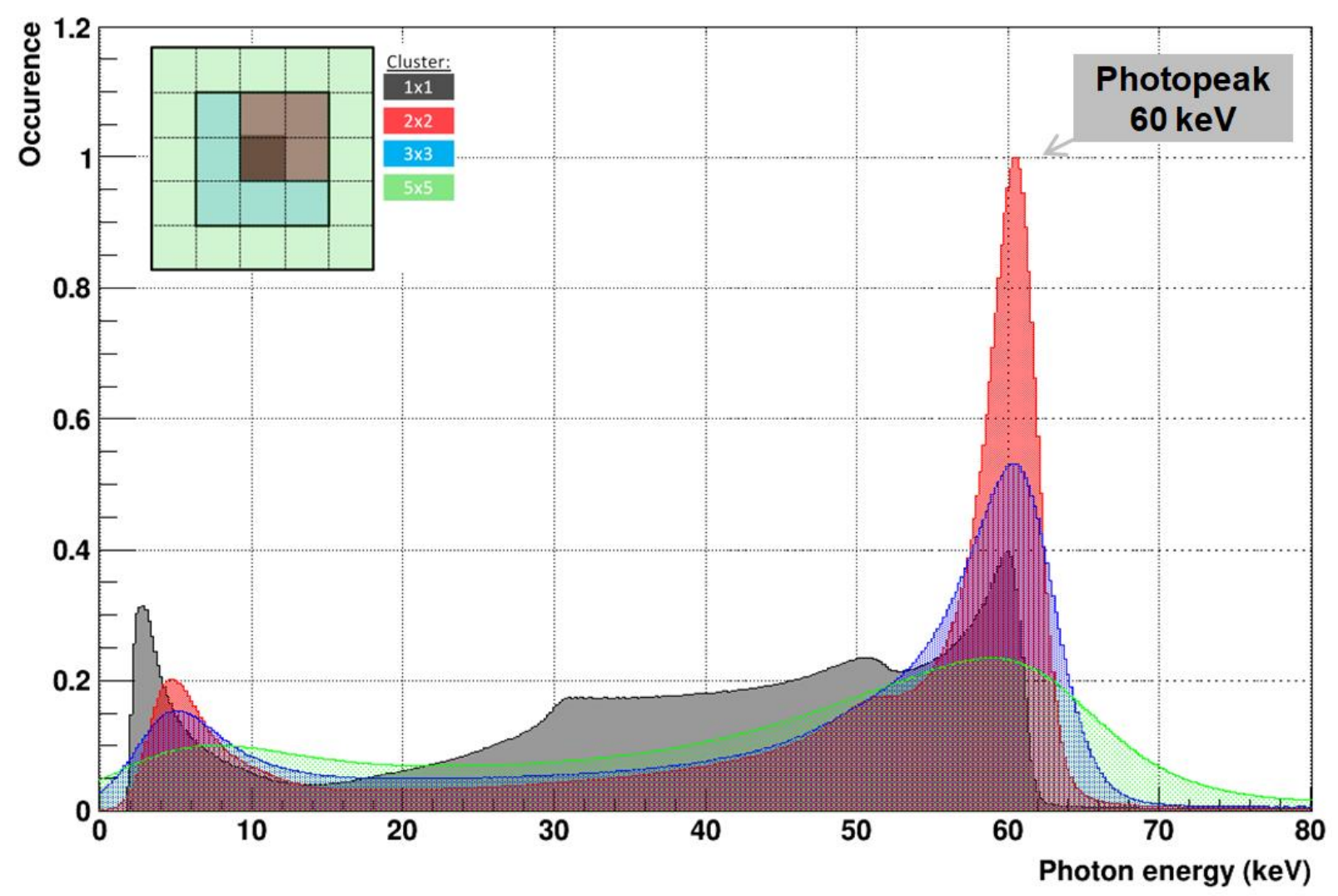

Figure 6: Energy spectra of a GaAs:Cr/JUNGFRAU assembly being illuminated with monochromatic photons of 60 keV using different number of pixels for the energy reconstruction (1x1 cluster spectrum: black | 2x 2 cluster spectrum: red | $3 \times 3$ cluster spectrum: blue | 5x5 cluster spectrum: green). The integration time is $2 \mu$ s and the bias voltage $-\mathbf{3 0 0} \mathrm{V}$. The energy resolution (FWHM) of the photopeak of the $2 \times 2$ cluster spectrum (red) is $4.14 \mathrm{keV}$ or $6.9 \%$. (The edge in the $1 \times 1$ cluster spectrum at around $2 \mathrm{keV}$ is the noise cut-off of the cluster finder.)

\section{Charge transport properties}

The Hecht relationship describes the dependency of the induced signal produced by a constant number of charge carriers as function of the applied sensor bias voltage. The $(\mu \cdot \tau)$ product of charge carriers of one polarity can be determined by injecting the charge carriers close to the backside electrode [20],[21]. Fluorescence photons from molybdenum were used, predominately emitting photons with an energy of 17.4 $\mathrm{keV}$ (weighted mean energy of $\mathrm{K}_{\alpha 1}$ and $\mathrm{K}_{\alpha 2}$ ). The mean penetration depth for photons with this energy is around $31 \mu \mathrm{m}$ in GaAs, ensuring a shallow absorption profile close to the backside contact and thus a complete drift of the charge carriers through the sensor.

Hamann proposes a modified Hecht relationship taking into account charge trapping and the small pixel effect [2] (eq.2). The approximation divides the sensor into two parts: In the first region, no charge is induced while drifting as the weighting potential is negligible and only charge trapping occurs. Only in the second region, typically having the thickness of the pixel pitch, do drifting charge carriers induce charge on the electrodes (The electric field in this region is approximated to be linear.) 
Equation 2: Modified Hecht relationship. ( $\mathrm{N}_{0}$ : Initially deposited number of charge carriers, q: Elementary charge, $d$ : Sensor thickness, D: Thickness of sensor, where charge is induced (approximated as pixel pitch in depth), U: Sensor bias voltage, $\mathrm{U}_{0}$ : Minimum voltage to induce signal)

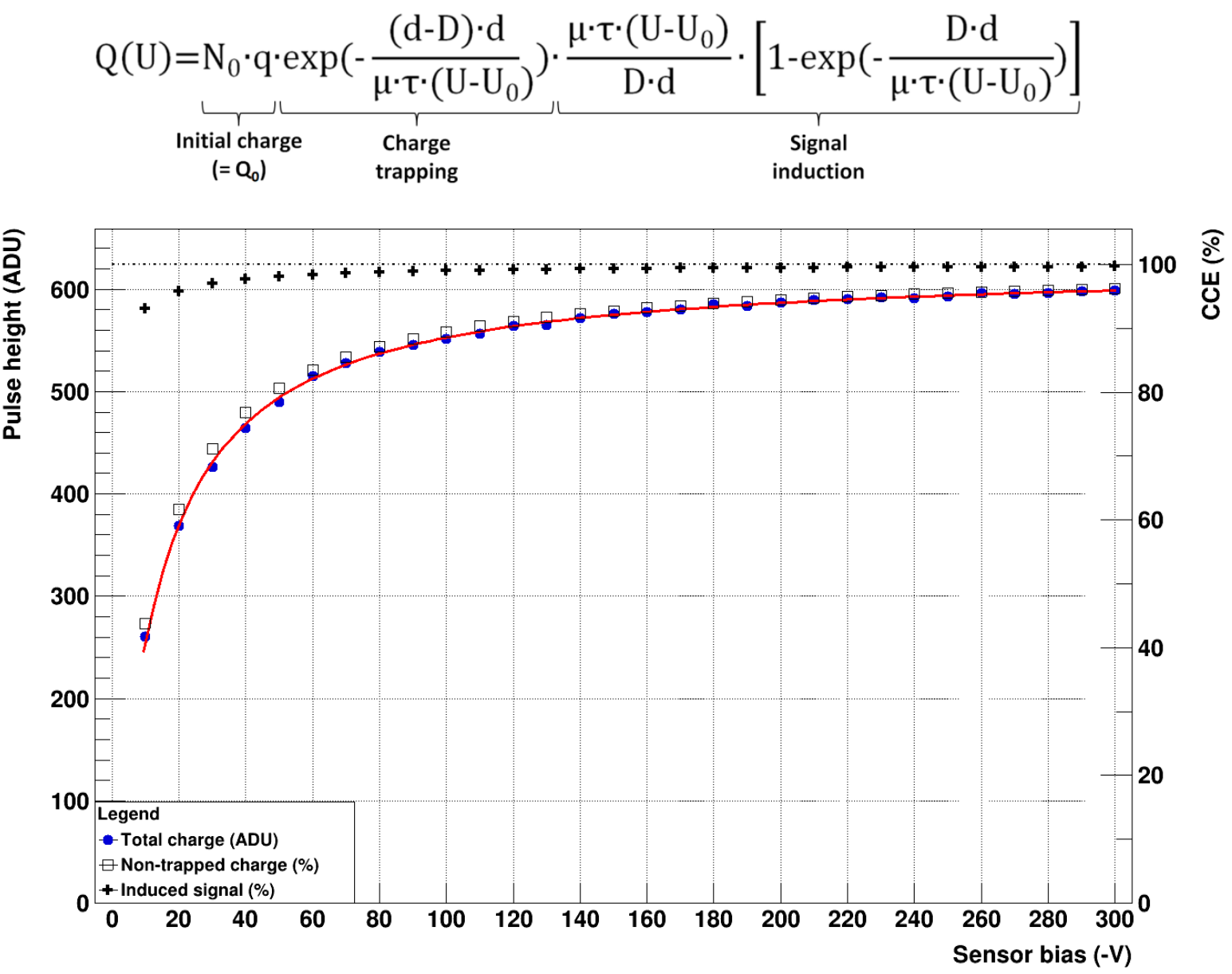

Figure 7: Hecht curve of a GaAs:Cr sensor using molybdenum fluorescence photons with an energy of $17.4 \mathrm{keV}$. The data was fitted with equation 2 (red solid line). The $(\mu \cdot \tau)_{\mathrm{e}}$ is $(1.831 \pm 0.002) \cdot 10^{-4} \mathrm{~cm}^{2} / \mathrm{V}$ and the CCE at $-300 \mathrm{~V}$ is $(95.97 \pm 0.01) \%$. The solid blue points represent the measured pulse height, the white squares indicate the relative amount of non-trapped charge carriers reaching the charge induction region and the black symbols show the relative amount of charge induced by the charge carriers reaching the charge induction region.

Fig. 7 shows the pulse height of the $17.4 \mathrm{keV}$ photons as function of the applied bias voltage. The data was fitted using equation 2 . The $(\mu \cdot \tau)_{e}$ product is $(1.831 \pm 0.002) \cdot 10^{-4} \mathrm{~cm}^{2} / \mathrm{V}$ which is in reasonable agreement with results published by other authors in the range between $(0.75-1.75) \cdot 10^{-4} \mathrm{~cm}^{2} / \mathrm{V}[2],[22],[23],[24]$. The charge collection efficiency (CCE) was calculated by taking the ratio between saturation value of the Hecht function $Q_{0}$ and the pulse height at the respective sensor bias voltage. The CCE at the maximum sensor bias voltage of -300 $V$ is $(95.97 \pm 0.01) \%$. By using the values for $(\mu \cdot \tau)_{e}$ and $N_{0}$ from the fit, the contributions from the trapping region and the charge induction region were calculated individually for different sensor bias voltages. According to these findings the charge loss and therefore also the reduction of the CCE is clearly dominated by charge trapping while drifting towards the charge induction region.

When reversing the polarity to measure the $(\mu \cdot \tau)$ product of the holes, no signal could be measured, presumably due to the short hole lifetime in GaAs: $\mathrm{Cr}[19],[22],[25]$. 


\section{'Crater effect'}

The 'crater effect' describes the effect that some photon hits result in a negative sum of the pulse heights in the pixels surrounding the photon hit. This effect was observed during measurements with the GaAs:Cr sensor, especially when using photon energies above $30 \mathrm{keV}$. Fig. 8 shows a random example of the pedestal and gain corrected signals of part of the pixel matrix of a GaAs: $\mathrm{Cr}$ sensor being irradiated with $60 \mathrm{keV}$ photons. The pixel cluster IV clearly shows this 'crater effect'.

In order to further investigate this effect, each photon hit was categorized by using the sum of the signal in the adjacent pixels around the pixel with the highest signal:

A - Charge sharing: The sum of the signals in the eight neighbors around the pixel with the highest signal is three times higher than the noise of these eight pixels, indicating that a significant amount of charge is shared among several pixels (Fig. 8, top, Cluster I+II).

B - Central hit: The sum of the signals in the eight neighbors around the pixel with the highest signal is within a window of plus/minus three times of the noise of these eight pixels, indicating that the majority of charge is collected in the central pixel and no crater is present (Fig. 8, top, Cluster III).

$\mathrm{C}$ - Crater hit: The sum of the signals in the eight neighbors around the pixel with the highest signal is more negative than minus three times the noise of these eight pixels (Fig. 8, top, Cluster IV).

The dataset of the irradiation with $60 \mathrm{keV}$ monochromatic photons was taken with a test system consisting of three sensors (1x GaAs:Cr sensor, 1x CdTe sensor, $1 x$ silicon sensor) which were operated in parallel. The integration time was $2 \mu$ s and the sensor bias voltage was $-300 \mathrm{~V}$ for the GaAs sensor, $-500 \mathrm{~V}$ for the CdTe sensor and $+240 \mathrm{~V}$ for the silicon sensor.

The crater effect can be visualized by a correlation plot showing the detected energy in the central pixel on the $x$ axis and the summed energy of the eight neighboring pixels on the y-axis (Fig.8, bottom, left and right). The horizontal black dashed lines indicate the three times noise level of the eight neighboring pixels which was used a criteria to define a 'crater hit', i.e. a photon hit where the crater effect is present.

The presence of crater hits is visible in region $\mathrm{C}$ of the correlation plot of the GaAs: $\mathrm{Cr}$ sensor (Fig. 8, bottom left). The sum of the signals of the eight neighbors is reaching negative values of more than $40 \%$ of the photon energy. For very negative values the signal in the central pixel is reduced, as can be seen in the example (Fig. 8, top, Cluster IV). Such a crater effect was not visible in the CdTe (Fig. 8, bottom right). 

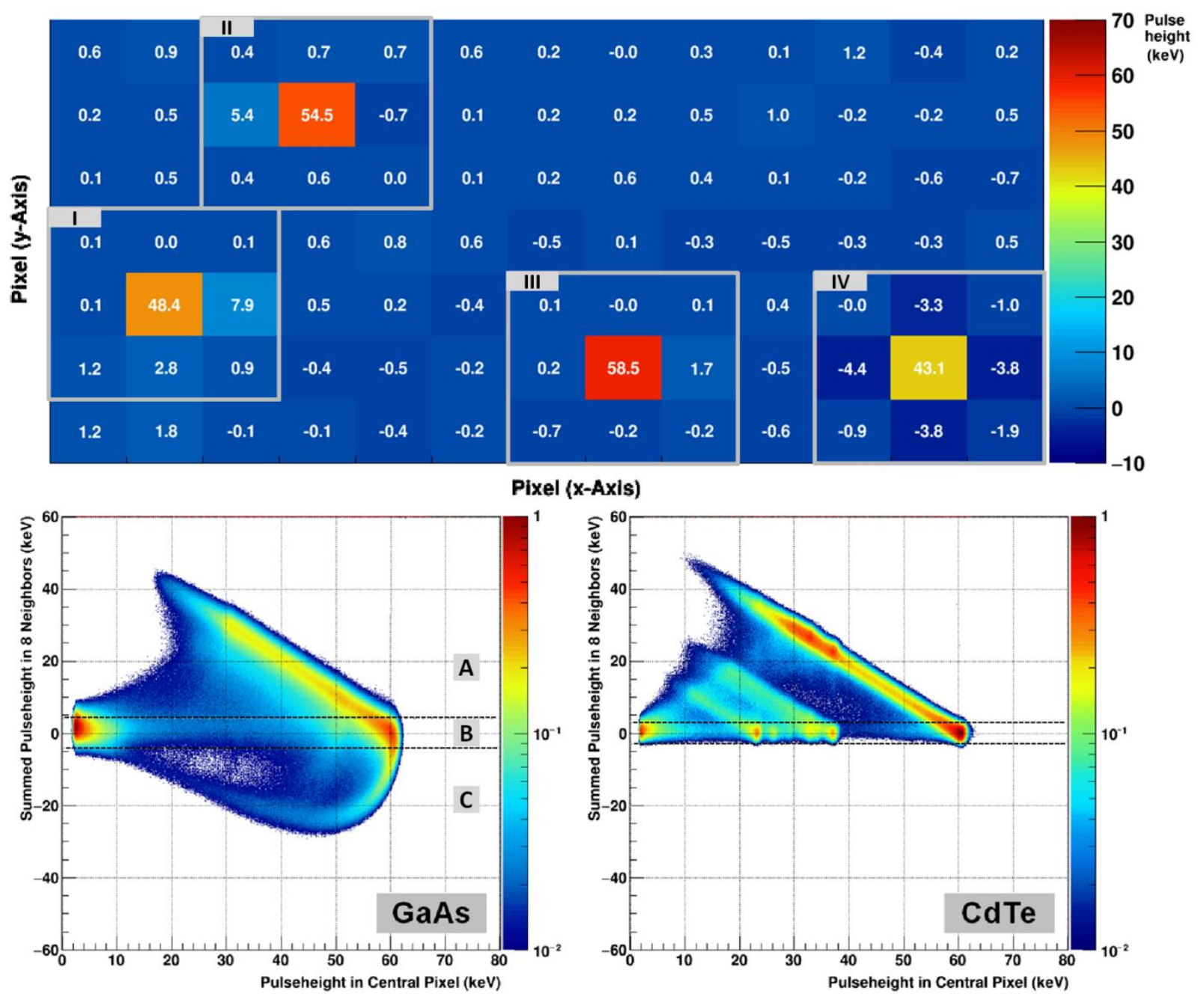

Figure 8: (top) Example of monochromatic $60 \mathrm{keV}$ photon hits on a fraction of the pixel matrix (gain and pedestal corrected) in units of keV. Grey outlines mark $3 \times 3$ pixel clusters centered around the pixel with the highest signal. Cluster I and II are clusters where the charge is shared among several pixels (Cluster I: Central pixel: 48.4 keV, $3 \times 3$ cluster: 61.5 keV, Charge in neighbors: $13.1 \mathrm{keV}$ | Cluster II: Central pixel: $54.5 \mathrm{keV}, 3 \times 3$ cluster: $62.0 \mathrm{keV}$, Charge in neighbors: $7.5 \mathrm{keV}$ ). Cluster III is assigned a 'central hit' with more than $98 \%$ of the charge in the center (Cluster III: Central pixel: 58.5 keV, $3 \times 3$ cluster: 59.5 keV, Charge in neighbors: $1.0 \mathrm{keV}$ ). Cluster IV is a crater hit, with a strong negative signal in the pixel around the central pixel

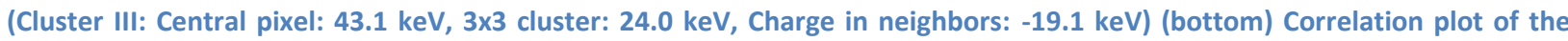
pulse height in the central pixel (x-axis) as function of the summed pulse heights of its eight neighbors (y-axis) for $60 \mathrm{keV}$ monochromatic photons. A: Charge shared photon hits, B: Central hits, C: Crater hits (left) GaAs:Cr sensor (right) CdTe sensor

Low flux fluorescence measurements have been performed using various fluorescence targets ( $\mathrm{Csl}, \mathrm{W}$ and $\mathrm{Pb}$ ) which emits photons in a wide energy range. Each identified photon hit was again classified according to the sum of the pulse heights in the eight adjacent pixels (Tab. 2). The probability to observe a crater hit (case C) increases with increasing photon energy and reaches up to $17.1 \%$ for the highest photon energy. Possibly this is due to the different photon absorption profiles, where higher energetic photons reach further into the sensor (d $\mathrm{cs}_{\alpha 1}=147.3 \mu \mathrm{m}, \mathrm{dw}_{\alpha 1}=891.3 \mu \mathrm{m}, \mathrm{dpb}_{\alpha 1}=1912.0 \mu \mathrm{m}$ ) [10],[11].

The distribution of the negative sum of pulse heights of the eight neighbor pixels is a continuous spectrum with a shoulder at roughly $-40 \%$ of the most probable impinging photon energy from the fluorescence targets (Fig. 9 , left) [30]. When plotting a distribution map of the crater hits, no spatial pattern or preference can be seen, indicating that the crater effect does not originate from defect structures in the crystal (Fig. 9, right). 
Table 1: Classification of the events for different photon energies. (Deviation from $100 \%$ are due to rounding errors).

\begin{tabular}{|l|c|c|c|}
\hline & $\begin{array}{c}\text { Csl } \\
\left(K_{\alpha 1, c s}=31.0 \mathrm{keV} / \mathrm{K}_{\alpha 1, \mathrm{I}}=28.6 \mathrm{keV}\right)\end{array}$ & $\begin{array}{c}\text { W } \\
\left(\mathrm{K}_{\alpha 1}=59.3 \mathrm{keV}\right)\end{array}$ & $\begin{array}{c}\text { Pb } \\
\left(\mathrm{K}_{\alpha 1}=79.2 \mathrm{keV}\right)\end{array}$ \\
\hline A - Charge shared event & $68.7 \%$ & $56.7 \%$ & $55.6 \%$ \\
\hline B - Central hit event & $28.1 \%$ & $\mathbf{2 9 . 2 \%}$ & $27.4 \%$ \\
\hline C - Crater hitevent & $\mathbf{3 . 2 \%}$ & $\mathbf{1 4 . 0 \%}$ & $\mathbf{1 7 . 1 \%}$ \\
\hline
\end{tabular}
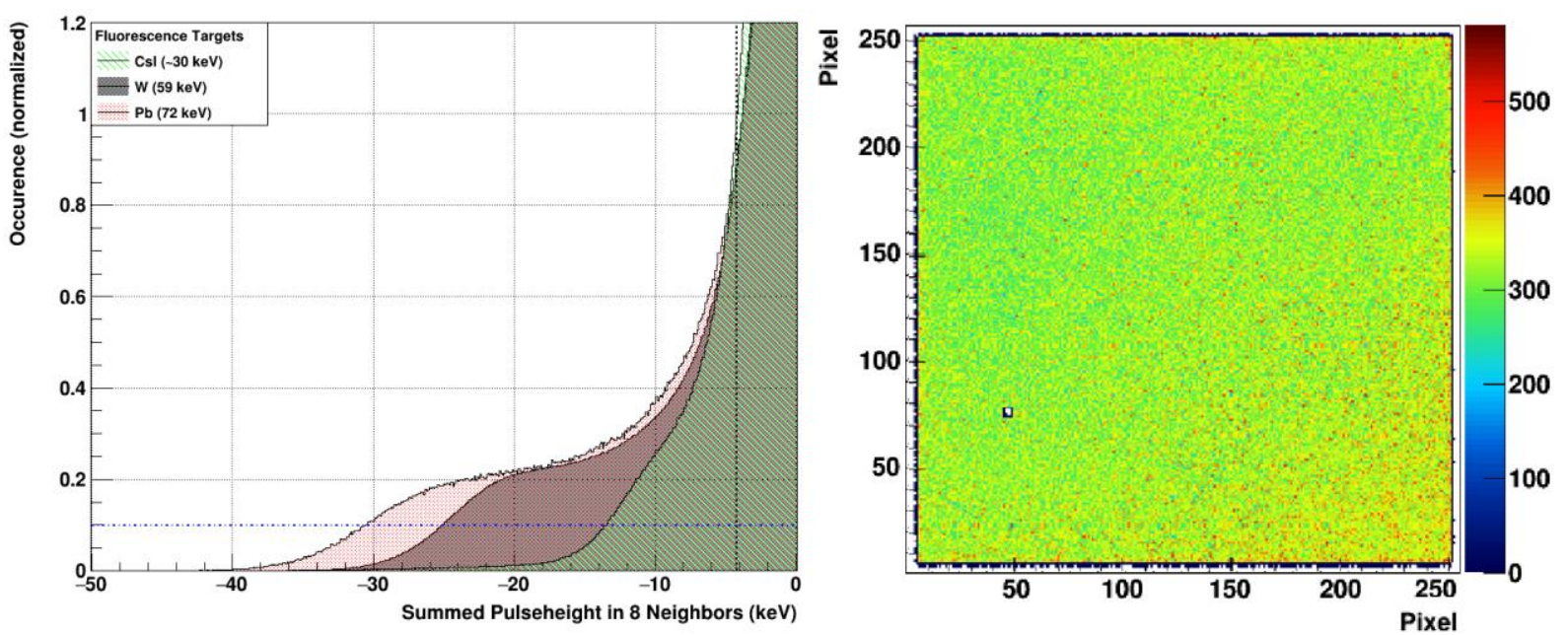

Figure 9: (left) Histogram of the summed negative pulseheight of the eight direct neighbors around a central hit for three different incident photon energies (Csl, W, Pb). (right) Occurrence map of crater hits. Note that the chip edges are excluded by the cluster finder.

Such a behavior was described earlier by other authors and assigned to the short lifetime of the non-collected charge carriers [26]. In agreement with these findings, the short lifetime of the holes in GaAs: $\mathrm{Cr}$ (which also has been mentioned previously in literature [19][22][25]) could be responsible for the observed crater effect: When a photon is absorbed close to the pixel electrode, a negative signal will be induced on the neighboring electrodes by the electrons drifting to the collecting electrode. Typically this negative signal is compensated by charge carriers of the opposite polarity (holes) drifting to the backside electrode. However, the short lifetime of the holes (in combination with the short integration time of the measurements of $t_{i n t}=2 \mu \mathrm{s}$ ) prevents the holes from drifting to the backside electrode and an overall negative signal remains on the adjacent pixel(s).

In order to gain a better understanding of the crater effect, simulations have been performed. The simulation considered the following processes: X-ray photon absorption (including fluorescence photons) and carrier generation, drift and diffusion (DD) of electrons and holes with constant diffusion coefficients, charge trapping, as well as signal formation at the readout electrodes using a weighting potential/field calculated, based on W. Riegler [27]. In addition to the simulated pulse height, white noise has been added. Simulations with different combinations of mobility in DD model and lifetimes in trapping model (for holes and electrons) taking into account the results from the Hecht measurements for the values for electrons, have been done. The simulated datasets were treated with exactly the same analysis script which was used for the analysis of the measured data.

The best agreement between simulated and measured data was obtained for a hole lifetime of $\tau_{h}=1.4 \mathrm{~ns}$ and by using the parameters presented in table 2. Fig. 10 shows a direct comparison between the simulated and experimental data. Fig. 11 shows results from simulated data, where the lifetime of the holes has been varied between $\tau_{h}=1.4 \mathrm{~ns}$ up to $\tau_{h}=100 \mathrm{~ns}$. The negative signal in the neighboring pixels is reducing with increasing lifetimes of the holes. As it is difficult to measure carrier lifetimes that are far shorter than the charge collection times directly, comparing the negative swing in the correlation plot with simulated data can potentially be an interesting tool to determine such short charge carrier lifetimes in the nanosecond regime. 
Table 2: Overview of the mobility and lifetime values from the simulations which yielded the best agreement between simulated and measured data. (In order to reduce the parameter space of the simulations, one condition for the simulations was that the product of mobility and lifetime of the electrons has to match the value previously obtained by the Hecht measurements $( \pm 20 \%)$. As no $\mu \cdot \tau$ product could be measured for holes, the parameter space for the holes was not confined.)

\begin{tabular}{|c|c|c|}
\hline & Mobility & Lifetime \\
\hline Electrons & $2585 \mathrm{~cm}^{2} / \mathrm{V} \cdot \mathrm{s}$ & $80 \mathrm{~ns}$ \\
\hline Holes & $171 \mathrm{~cm}^{2} / \mathrm{V} \cdot \mathrm{s}$ & $1.4 \mathrm{~ns}$ \\
\hline
\end{tabular}
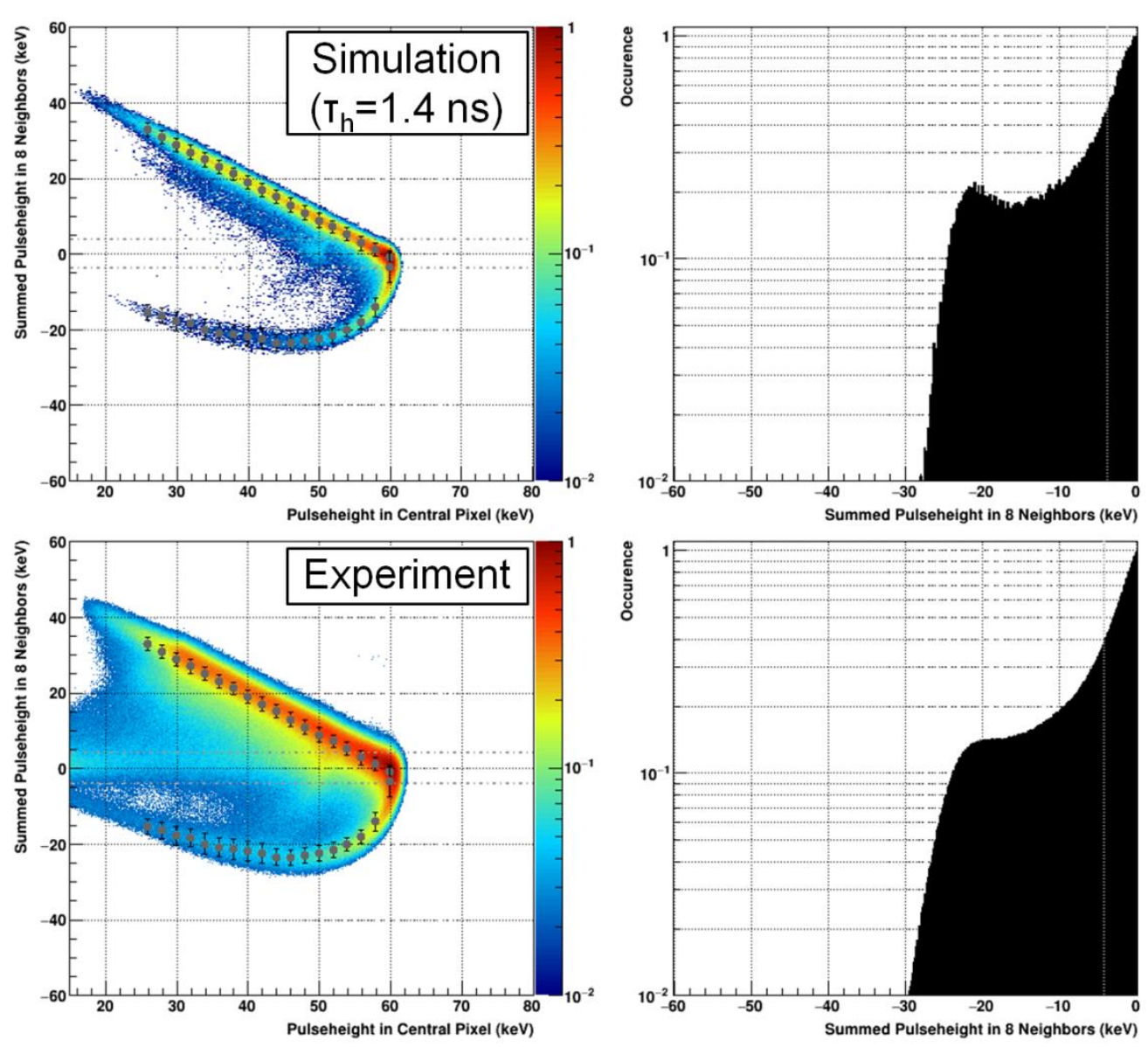

Figure 10: (left) Correlation plot of the signal in the central pixel (x-axis) and the summed signal in the eight neighboring pixels (y-axis). The simulated data (top) is nicely able to reproduce the negative undershoot which can be seen in the measured data (bottom). (right) Projection of the negative signal in the eight neighboring pixels. 

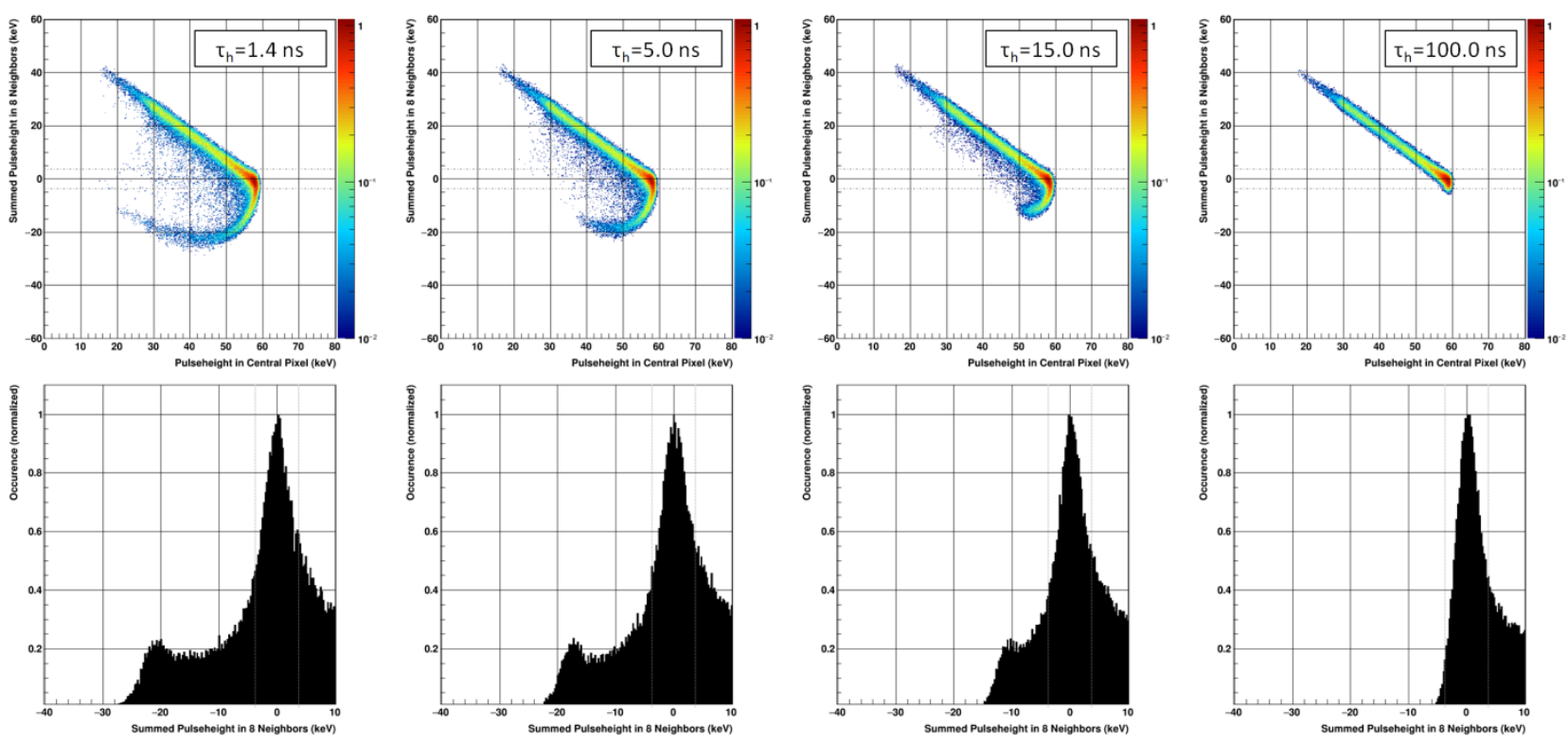

Figure 11: Comparison of the results from the simulation for different values of hole lifetimes (from left to right: $\tau_{\mathrm{e}}=1.4 \mathrm{~ns} /$ $5.0 \mathrm{~ns} / 15.0 \mathrm{~ns} / \mathbf{1 0 0 . 0} \mathrm{ns}$ ). In the top row, correlation plots of the signal in the central pixel (x-axis) and the summed signal in the eight neighboring pixels (y-axis) are shown. In the bottom row, the pulse height spectra of the eight neighboring pixels are drawn. The effect of a negative signal in the neighboring pixels is reducing with increasing lifetime of the holes. (Note: Fluorescence photons have been neglected in this dataset.)

The crater effect has severe implications for the operation of the detector. Fig. 12 (bottom) shows a modified version of the (monochromatic) $60 \mathrm{keV}$ energy spectra shown above (Fig. 6), taking into account only crater hits. The single pixel spectrum ( $=1 \times 1$ cluster) shows that the energy in the central pixel is typically properly measured with a tail towards lower energies. The low energy tail arises when photons are absorbed very close to the readout electrode (=few tens of microns), resulting in a reduced signal height as the electron contribution of the overall signal is not dominating and the complementary hole signal to achieve a full signal is missing due to the aforementioned short lifetime of the holes. The spectral information starts to smear out when more pixels are used for the energy reconstruction. Already when taking into account $2 \times 2$ clusters for the energy reconstruction the photopeak is shifted towards lower energies. In the $3 \times 3 / 5 \times 5$ pixel cluster spectra no photopeak is visible anymore. The consequence of this observation is that charge reconstruction is not usable when a crater hit is present.

In contrary to charge integrating ASICs, the crater effect is not visible in single photon counting readout chips, which only compare the signal pulse height with a comparator threshold, although present as it is a sensor effect. In the case of using single photon counting detectors in the region around a crater hit the signal of simultaneously incoming photons will be reduced. This leads to a reduction of the measured number of photons at high photon rates. 

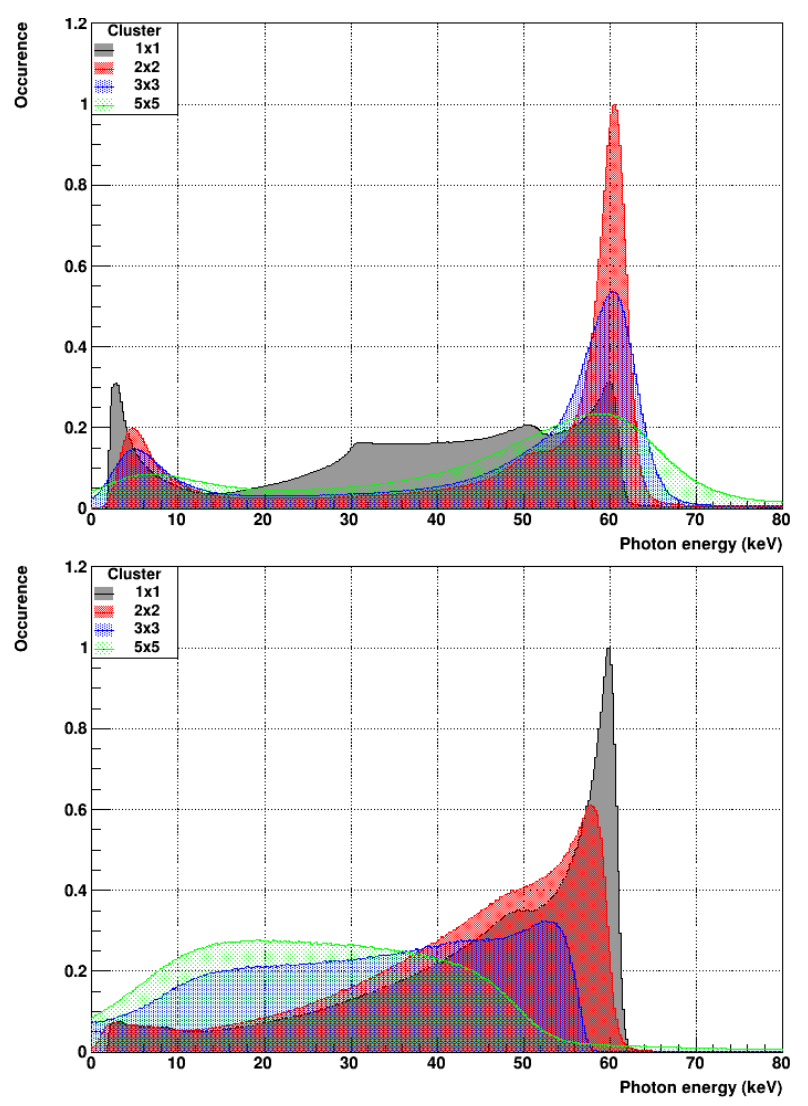

Figure 12: Energy spectra of monochromatic $60 \mathrm{keV}$ photons using different cluster sizes (1x1 cluster: black | 2x2 cluster: red | 3x3 cluster: blue | 5x5 cluster: green). (top) Measured spectra using only events, where the sum of the eight neighboring pixels was bigger than minus three times the noise of these eight pixels. (bottom) Measured spectra only taking into account events where the sum of the eight neighboring pixels was smaller than minus three times the noise of these eight pixels (= crater event). In case of crater events the charge in the central pixel is conserved (black spectrum), however the signal information is distorted when summing up pixels.

\section{Summary}

Various results from the characterization of $\mathrm{GaAs}: \mathrm{Cr}$ sensors on the charge-integrating readout chip JUNGFRAU have been presented.

The dark current through the sensor was determined to be $391.0 \mathrm{pA}$ per pixel or $25.1 \mu \mathrm{A}$ for the whole $256 \times 256$ pixel matrix at $\mathrm{U}=-300 \mathrm{~V}$ and at $\mathrm{T}=+15^{\circ} \mathrm{C}$. That translates to a resistivity of the GaAs:Cr sensors of $\rho=8.5 \cdot 10^{8} \Omega \cdot \mathrm{cm}$. This relatively high dark current restricts the integration times to a maximum of a few $\mu \mathrm{s}$, limiting the potential use cases at synchrotron sources. The dark current can be reduced by a factor of 9 by cooling the sensor to $-12{ }^{\circ} \mathrm{C}$, thus allowing for longer integration times.

The gain dispersion over the pixel matrix is around $3.29 \%$ (r.m.s.) and the noise performance corresponds to 107.8 $\mathrm{e}^{-} \mathrm{ENC}$ (r.m.s.) at $2 \mu \mathrm{s}$ integration time. The energy resolution (FWHM) of monochromatic $60 \mathrm{keV}$ photons is 4.14 $\mathrm{keV}$ or $6.9 \%$. The charge carrier transport properties of the electrons were determined by a measurement using a modified Hecht relation taking into account the small pixel effect and charge trapping: The $(\mu \cdot \tau)$ e product is $(1.831$ $\pm 0.002) \cdot 10^{-4} \mathrm{~cm}^{2} / \mathrm{V}$ and the charge collection efficiency at the maximum sensor bias voltage of $-300 \mathrm{~V}$ is (95.97 \pm $0.01) \%$.

The short lifetime of holes in GaAs:Cr is the reason for negative signals in pixels adjacent to the collecting electrode, when the photon is absorbed close to the readout electrodes. The sum of the negative pulse height is a continuous spectrum with a shoulder at approximately $-40 \%$ of the incoming photon energy. By means of 
simulations this crater effect was understood as originating from the short lifetime of the holes which are not able to compensate the negative charge induced on the adjacent pixel(s), when the photon is absorbed close to the pixel electrode. The charge carriers transport properties which were extracted from simulations yielded $\mu_{\mathrm{e}}=2585$ $\mathrm{cm}^{2} / \mathrm{V} \cdot \mathrm{s}$ and $\tau_{\mathrm{e}}=80 \mathrm{~ns}$ for electrons and $\mu_{\mathrm{h}}=171 \mathrm{~cm}^{2} / \mathrm{V} \cdot \mathrm{s}$ and $\tau_{\mathrm{h}}=1.4 \mathrm{~ns}$ for holes. The probability to observe such an event is increasing with increasing photon energy as the probability to absorb a photon close to the readout electrodes increases. It can reach up to $17.1 \%$ in case of irradiation with fluorescence photons of $\mathrm{Pb}\left(\mathrm{K}{ }_{\alpha 1}=79.2\right.$ $\mathrm{keV})$. A rough approximation then yields that craters are created in a $88 \mu \mathrm{m}$ thick layer in front of the readout electrodes.

The crater effect is a sensor effect and therefore also present in single photon counting ASICs, although not directly visible. However, it leads to reduced measured count rates at high incoming photon rates due to an effective increase of the counter threshold when a crater is present.

\section{References}

[1] Berger, M.J., Hubbell, J.H., Seltzer, S.M., Chang, J., Coursey, J.S., Sukumar, R., Zucker, D.S., and Olsen, K., XCOM: Photon Cross Section Database (version 1.5), [Online] Available: http://physics.nist.gov/xcom, National Institute of Standards and Technology, Gaithersburg, MD, USA (2010)

[2] E. Hamann, Characterization of high resistivity GaAs as sensor material for Photon Counting Semiconductor Pixel Detectors, Ph.D. thesis, Albert-Ludwigs-University Freiburg, Germany (2013)

[3] A. Cola, I. Farella, The polarization mechanism in CdTe Schottky detectors, Appl. Phys. Lett. 94, 102113, https://doi.org/10.1063/1.3099051 (2009)

[4] D. Greiffenberg, Charakterisierung von CdTe-Medipix2-Pixeldetektoren, Ph.D. thesis, Albert-Ludwigs-University Freiburg, Germany (2010)

[5] P. Sellin, Recent advances in compound semiconductor radiation detectors, Nucl. Instr. And Meth. A, Vol. 513, pp. 332-339, https://doi.org/10.1016/j.nima.2003.08.058 (2003)

[6] P. Zambon et al., Spectral response characterization of CdTe sensors of different pixel size with the IBEX ASIC, Nucl. Instr. And Meth. A, Vol. 892, pp. 106-113, https://doi.org/10.1016/j.nima.2018.03.006 (2018)

[7] J. Becker et al., Characterization of chromium compensated GaAs as an X-ray sensor material for chargeintegrating pixel array detectors, J. Instrum. 13, P01007, https://doi.org/10.1088/1748-0221/13/01/P01007 (2018)

[8] D. Pennicard et al., LAMBDA 2M GaAs - A multi-megapixel hard X-ray detector for synchrotrons, J. Instrum. 13, C01026, https://dx.doi.org/10.1088/1748-0221/13/01/C01026 (2018)

[9] C. Ponchut et al., Characterization of GaAs:Cr pixel sensors coupled to Timepix chips in view of synchrotron applications, JINST 12 C12023 (2017)

[10] X-ray data booklet, Third edition, Lawrence Berkeley National Laboratry, http://xdb.Ibl.gov (2009)

[11] M.J. Berger et al., XCOM: Photon Cross Section Database (version 1.5), National Institute of Standards and Technology, Gaithersburg, MD, http://physics.nist.gov/xcom (2010)

[12] W. R. Harding et al., Gallium Arsenide for $\gamma$-Ray Spectroscopy, Nature 187, 405, doi:10.1038/187405a0 (1960) 
[13] G. I. Ayzenshtat et al., GaAs resistor structures for X-ray imaging detectors, Nucl. Instr. And Meth. A, 487, p. 96 -101 (2002)

[14] A.V. Tyazhev et al., GaAs radiation imaging detectors with an active layer thickness up to $1 \mathrm{~mm}$, Nucl. Instr. And Meth. A, 509, p. $34-39$ (2003)

[15] L. Tlustos, G. Shelkov, O. P. Tolbanov, Characterisation of a GaAs(Cr) Medipix2 hybrid pixel detector, Nucl. Instr. And Meth. A, 633, p. 103-107 (2011)

[16] A. Mozzanica et al., The JUNGFRAU Detector for Applications at Synchrotron Light Sources and XFELs, Synchrotron Radiation News, 31:6, pp. 16-20, doi: 10.1080/08940886.2018.1528429 (2018)

[17] A. Mozzanica et al., Characterization results of the JUNGFRAU full scale readout ASIC, JINST 11 C02047 (2016)

[18] BM05 Characteristics,

http://www.esrf.eu/files/live/sites/www/files/UsersAndScience/Experiments/XNP/BM05/BM05characteristics.pdf (2017)

[19] M. Veale, Chromium compensated gallium arsenide detectors for $X$-ray and $\gamma$-ray spectroscopic imaging, Nucl. Instr. And Meth A 752, pp.6-14, doi: 10.1016/j.nima.2014.03.033 (2014)

[20] K. Hecht, Zum Mechanismus des lichtelektrischen Primärstromes in isolierenden Kristallen, Zeitschrift für Physik A Hadrons and Nuclei, Vol. 77, No. 3-4 (1932)

[21] D. Greiffenberg, Energy resolution and transport properties of CdTe-Timepix-Assemblies, JINST 6 C0105, doi:10.1088/1748-0221/6/01/C01058 (2011)

[22] G. Ayzenthat, The formation of amplitude spectra in X-ray pixel detectors made of gallium arsenide, Journal of X-Ray Science and Technology, vol. 25, no. 4, pp. 585-595, doi: 10.3233/XST-16136 (2017)

[23] P. Smolzanskiy, Properties of GaAs:Cr-based Timepix detectors, JINST 13, T02005, https://doi.org/10.1088/1748-0221/13/02/T02005 (2018)

[24] I. Chsherbakov, Electron mobility-lifetime and resistivity mapping of GaAs:Cr wafers, JINST 13, C02016, https://doi.org/10.1088/1748-0221/12/02/C02016 (2017)

[25] A. Zarubin, Non-equilibrium Charge Carriers Life Times in Semi-Insulating GaAs Compensated with Chromium, $7^{\text {th }}$ International Workshops and Tutorials on Electron Devices and Materials, pp. $345-348$, doi: 10.1109/SIBEDM.2006.231988 (2006)

[26] J. D. Eskin, H. H. Barrett, H. B. Barber, Signals induced in semiconductor gamma-ray imaging detectors, Journal of Applied Physics 85, 647, https://doi.org/10.1063/1.369198 (1999)

[27] W. Riegler, G. Aglieri Rinella, Point charge potential and weighting field of a pixel or pad in a plane condenser, Nucl. Instr. And Meth. A 767, pp.267-270, https://dx.doi.org/10.1016/j.nima.2014.08.044 (2014) 